\title{
¿HAY DIFERENCIAS ENTRE LAS ACTITUDES HACIA LA CONDUCTA SEXUAL QUE TIENEN LAS PERSONAS CON DISCAPACIDAD INTELECTUAL Y LAS DE LOS PROFESIONALES QUE TRABAJAN CON ELLOS?
}

\author{
Vicente Morell-Mengual \\ Departamento de Psicología Evolutiva y de la Educación \\ Universitat de València \\ vicente.morell@uv.es \\ M. D. Gil-Llario \\ Departamento de Psicología Evolutiva y de la Educación \\ Universitat de València \\ Olga Fernández-García \\ Departamento de Psicología Evolutiva y de la Educación \\ Universitat de València \\ Beatriz Gil-Juliá \\ Departamento de Personalidad, Evaluación y Tratamientos Psicológicos \\ Universitat de València \\ Yarisa Nicola \\ Departamento de Psicología Evolutiva y de la Educación \\ Universitat de València
}

Recepción Artículo: 14 Febrero 2020

Admisión Evaluación: 4 marzo 2020

Informe Evaluador 1: 18 marzo 2020

Informe Evaluador 2: 20 Marzo 2020

Aprobación Publicación: 20 abril 2020

\section{RESUMEN}

Las actitudes hacia la sexualidad constituyen uno de los elementos cognitivo-emocionales más determinantes del funcionamiento sexual. En este sentido, la sexualidad de las personas con discapacidad intelectual (DI) está fuertemente influenciada por diversos mitos, estereotipos y prejuicios que repercuten en la perpetuación de ciertas actitudes negativas que limitan sus vivencias sexuales. El objetivo de este estudio es analizar las actitudes hacia la conducta sexual de las personas con discapacidad intelectual que poseen las propias personas que integran este colectivo y los profesionales que trabajan con ellos. Para ello, se utiliza la Escala de percepción sexual para personas con discapacidad intelectual en una muestra de 300 personas con DI leve o moderada con edades comprendidas entre 19 y 55 años y la Escala de Actitudes hacia la sexualidad en personas con discapacidad intelectual en 100 profesionales con edades comprendidas entre 20 y 66 años. Los resultados indican que el $36.4 \%$ de las personas con DI poseen actitudes conservadoras, el $55.3 \%$ actitudes moderadas y el $8.3 \%$ actitudes liberales. Por su parte, el $14 \%$ de los profesionales presentan actitudes moderadas y el $86 \%$ actitudes liberales. En general, los profesionales poseen actitudes significativamente más liberales en todas las conductas sexuales analizadas (besos, caricias y/o sexo oral), excepto en la práctica del coito vaginal donde son las personas con DI 


\section{¿HAY DIFERENCIAS ENTRE LAS ACTITUDES HACIA LA CONDUCTA SEXUAL QUE TIENEN LAS PERSONAS CON DISCAPACIDAD INTELECTUAL Y LAS DE LOS PROFESIONALES QUE TRABAJAN CON ELLOS?}

quienes presentan actitudes más erotofílicas. En conclusión, las personas con DI muestran actitudes mayoritariamente conservadoras a pesar de que los profesionales que trabajan con ellos son principalmente liberales. Y, por tanto, debería implementarse un módulo actitudinal en los programas de educación afectivo-sexual de las personas con DI, puesto que esta variable repercute significativamente en su salud sexual.

Palabras clave: actitudes; conducta sexual; discapacidad intelectual; profesionales

\section{ABSTRACT}

Are there differences between attitudes towards the behaviour sexuality of people with disabilities and those of the professionals who work with them? Attitudes towards sexuality are one of the most important cognitive-emotional determinants of sexual functioning. In this sense, the sexuality of persons with intellectual disabilities (ID) is influenced by myths, stereotypes and prejudices that encourage the perpetuation of certain negative attitudes and limit their sexual experiences. The aim of this study is to analyze the attitudes towards sexuality in people with intellectual disabilities and in professionals who work with them. For this purpose, we use a brief scale of attitudes towards sexual behaviour in a sample of 300 people with mild or moderate intellectual disability (ID), aged between 19 and 55 years old and 100 professionals, aged between 20 and 66 years old. Results reveal that $34.6 \%$ of people with ID have conservative attitudes, $55.3 \%$ moderate attitudes, and $8.3 \%$ liberal attitudes. On the other hand, $14 \%$ of the professionals present moderate attitudes and $86 \%$ liberal attitudes. In general, professionals have more liberal attitudes in all sexual behaviours (kissing, fondling and/or oral sex), except in vaginal intercourse where people with IDs have more erotophilic attitudes. In conclusion, people with ID report mostly conservative attitudes although professionals have mostly liberal attitudes. For this reason, an attitudinal module in affective-sexual education programs for people with ID should be implemented, because this variable has a significant impact on sexual health.

Keywords: attitudes; sexual behaviour; intellectual disability; professionals

\section{INTRODUCCIÓN}

La discapacidad intelectual (DI) constituye un trastorno del neurodesarrollo caracterizado por deficiencias en las funciones intelectuales, en resolución de problemas, pensamiento abstracto, planificación, juicio, razonamiento, aprendizaje a partir de la experiencia y aprendizaje académico; y deficiencias en el comportamiento adaptativo, que impiden la consecución de los estándares sociales y culturales para la independencia personal y la responsabilidad social y limitan el funcionamiento en una o más actividades de la vida diaria, tales como la participación social, la comunicación y la vida independiente (APA, 2013; OMS, 2019).

El grado de autonomía e independencia personal que tiene este colectivo ha variado significativamente según cada dimensión específica, desde una mayor permisividad en relación a los aspectos lúdicos, culturales laborales y/o deportivos (Álvarez, Vega, Spencer, González y Arriagada, 2019; Cezar, Figueiredo, Ferigato y Agostini, 2016; Pereira-Silva, Furtado y Andrade, 2018); a una mayor contención en relación a los aspectos relacionados con la sexualidad y la conducta sexual (Chou, Jane y Pu, 2015; Morell-Mengual, Gil-Llario, Díaz-Rodríguez y CaballeroGascón, 2017).

Las personas con discapacidad intelectual muestran los mismos intereses y necesidades sexuales que las personas sin discapacidad (Phasha y Runo, 2017) y así queda recogido en el artículo 25 de la Convención Internacional de Naciones Unidas sobre los Derechos de las Personas con Discapacidad "las personas con discapacidad tienen derecho a gozar del más alto nivel posible de salud sin discriminación por motivos de discapacidad, incluso en el ámbito de la salud sexual'. Aunque el desarrollo psicosexual de estas personas suele retrasarse unos 3 años con respecto a las etapas establecidas en el desarrollo normativo (Kijak, 2013), numerosas investigaciones refieren elevados porcentajes de actividad sexual. Nuestro equipo recientemente ha realizado un estudio donde se analizan diversos aspectos del comportamiento sexual en personas con DI leve o moderada (Gil-Llario, Morell-Mengual, Ballester-Arnal y Díaz-Rodríguez, 2018), encontrando que el 84\% ha mantenido relaciones sexuales de algún tipo con otra persona. Concretamente, las conductas más referidas son besos y caricias (99.2\%), coito vaginal (84.4) y sexo oral (80.3\%). 
Desafortunadamente, la sexualidad de las personas con DI sigue impregnada de diversos mitos, estereotipos y prejuicios que, a pesar de estar cada vez menos presentes en el imaginario colectivo, es necesario extinguir ya que promueven la perpetuación de algunas actitudes negativas que impiden y/o limitan la libre expresión de la sexualidad (Olavarrieta et al., 2013). Un estudio realizado por Siebelink, de Jong, Taal y Roelvink (2006) indica que las personas con DI tienen una actitud claramente positiva hacia los besos, los abrazos y las relaciones sexuales heterosexuales. Poseen, en promedio, una actitud casi neutral hacia las actividades sexuales de tipo impersonal (masturbación, visionado de películas para adultos y prostitución) y una actitud más conservadora con respecto a la homosexualidad. En contexto español, un trabajo que analiza las actitudes hacia la sexualidad que poseen un grupo de personas con DI determina que el $6.1 \%$ presenta actitudes conservadoras mientras que el 93.9\% tiene actitudes moderadas, no encontrando actitudes liberales o erotofílicas (Díaz-Rodríguez, Gil-Llario, Morell-Mengual, Salmerón-Sánchez y Ruiz-Palomino, 2016).

Las actitudes, valores y creencias hacia la sexualidad se construyen y mantienen en el marco de la familia, Ios amigos, la sociedad y la cultura (Morell-Mengual et al., 2017). En esta línea, algunos trabajados resaltan el impacto que ejercen las características individuales y sociales sobre las actitudes, sugiriendo que los aspectos culturales y el imaginario colectivo juega un papel determinante en la perpetuación del estigma hacia las personas con discapacidad intelectual (Ditchman, Easton, Batchos, Rafajko y Shah, 2017). Las actitudes no son inocuas e influyen en la adopción de comportamientos sexuales de riesgo. Algunas teorías clásicas como la Teoría Cognitiva Social (Bandura, 2012) o la Teoría de la Acción Razonada de Fishbein y Ajzen (1975) introducen las actitudes como variable mediadora de la conducta de riesgo. Específicamente, la Teoría Cognitiva Social postula que el aprendizaje se produce a través de un modelo y consiste en copiar o adoptar actitudes, creencias y valores observadas en una persona de referencia con la que uno mismo se identifica. Por su parte, la Teoría de la Acción Razonada establece que la intención conductual está fuertemente influenciada por dos variables: una personal referida a la actitud personal que consiste en la evaluación favorable o desfavorable por parte del sujeto de esa conducta, y otra de carácter social referida a las expectativas que el sujeto percibe de su ambiente socio-cultural, denominada norma subjetiva.

Los profesionales constituyen uno de los principales modelos de aprendizaje debido al tiempo que pasan junto a las personas con DI en los centros ocupacionales o en los recursos asistenciales. Por tanto, las actitudes que manifiestan los profesionales repercuten directamente sobre las actitudes que adoptan las personas con DI respecto a la ejecución de determinadas conductas. Debido al nivel de información y sensibilización que tienen Ios profesionales, las actitudes que poseen hacia la sexualidad de estas personas son, en general, bastante positivas (Bazzo, Nota, Soresi, Ferrari y Minnes, 2007; Meaney-Tavares y Gavidia-Payne, 2012). No obstante, existen estudios que indican actitudes desfavorables hacia la sexualidad fuertemente influenciados con los estereotipos de género tradicionales. Una investigación de Young, Gore y McCarthy (2012) que analiza las actitudes de los profesionales respecto a la sexualidad de personas con DI concluye que las mujeres son percibidas como sexualmente inocentes y los hombres como individuos muy interesados por la sexualidad.

Por ello, el presente estudio tiene como objetivo evaluar el grado de conservadurismo/liberalismo respecto a las actitudes hacia la conducta sexual, y averiguar si existen diferencias entre las actitudes tienen las personas con discapacidad intelectual y las de los profesionales que trabajan con ellos.

\section{MÉTODO}

\section{Muestra}

La muestra está conformada por 460 personas pertenecientes a dos colectivos, 360 personas con DI y 100 profesionales que trabajan con estas mismas personas. El grupo de personas con DI está compuesto por 180 hombres y 180 mujeres con edades comprendidas entre 19 y 55 años, siendo la edad media de 40 años ( $M=$ 39.97; DT = 15.50) y las edades más frecuentes, de 40 a 49 años (37.8\%) y de 21 a 29 años (20.3\%). Según el grado de discapacidad, el $41.1 \%$ tiene un nivel discapacidad leve $(n=140)$ y el $58.9 \%$ un nivel moderado. El 


\section{¿HAY DIFERENCIAS ENTRE LAS ACTITUDES HACIA LA CONDUCTA SEXUAL QUE TIENEN LAS PERSONAS CON DISCAPACIDAD INTELECTUAL Y LAS DE LOS PROFESIONALES QUE TRABAJAN CON ELLOS?}

$33.3 \%$ reside con su familia, el $33.3 \%$ en un centro residencial y el $33.3 \%$ en una vivienda tutelada. El grupo de profesionales está compuesto por 50 hombres y 50 mujeres con edades comprendidas entre los 22 y los 66 años, siendo las edades más frecuentes de 36 a 51 años (57\%). Por nivel educativo, el 36\% posee una titulación universitaria y el $63 \%$ estudios secundarios o de formación profesional.

\section{Instrumentos}

Cuestionario Ad-Hoc de Información Sociodemográfica. Este cuestionario recoge información sobre el sexo, la edad y el nivel educativo alcanzado (estudios secundarios, formación profesional o estudios universitarios.

AESXID. Escala de Actitudes hacia la sexualidad en personas con discapacidad intelectual (Gil-Llario, Fernández-García, Castro-Calvo, Caballero-Gascón y Ballester-Arnal). Instrumento compuesto por 18 ítems que evalúa actitudes hacia diversos aspectos de la sexualidad de personas con DI: intimidad, conducta sexual, habilidad para controlar los impulsos sexuales, etc. Se contesta mediante una escala tipo Likert con cinco opciones de respuesta que van desde 1 «completamente en desacuerdo» a 5 «completamente de acuerdo». Para este estudio únicamente se han utilizado tres ítems relacionados con la conducta sexual: besos y caricias «me parece bien que las personas con DI se besen o acaricien con otra persona», sexo oral «me parece bien que las personas con DI tengan relaciones sexuales siempre que no haya penetración» y coito vaginal «me parece bien que las personas con DI tengan relaciones sexuales con o sin penetración». Además de las puntuaciones en cada ítem, también se obtiene una puntuación total resultado de la suma de todas las respuestas, obteniendo un valor entre 3 y 15. Una mayor puntuación indica actitudes más erotofílicas respecto a la sexualidad. A efectos interpretativos se han establecido tres rangos: actitudes conservadoras 0 erotofóbicas de 3 a 6 puntos; actitudes moderadas de 7 a 11 puntos; y actitudes liberales 0 erotofílicas de 12 a 15 puntos.

POS. Escala de percepción sexual para personas con discapacidad intelectual (Scotti, Slack, Bowman y Morris, 1996; Díaz-Rodríguez, 2017). Escala compuesta por 28 ítems que evalúa las actitudes positivas o negativas en relación a diferentes comportamientos y prácticas sexuales en parejas tanto del mismo como de diferente sexo. Se responde mediante una escala de respuesta tipo Likert con cinco opciones que van desde 1 «nada aceptable» hasta 5 «muy aceptable». Para este estudio se han selecciona tres ítems relacionados con las siguientes prácticas sexuales: besos «beso prolongado con un compañero de diferente sexo», sexo oral «sexo oral con un compañero de diferente sexo» y coito vaginal "coito vaginal con un compañero de diferente sexo». Además de las puntuaciones en cada ítem, se calcula una puntuación global sumando todas las respuestas, con un valor entre 3 y 15. Una mayor puntuación indica actitudes más erotofílicas o liberales en relación a las diversas prácticas sexuales. A efectos interpretativos se han considerado tres rangos: actitudes conservadoras 0 erotofóbicas de 3 a 6 puntos; actitudes moderadas de 7 a 11 puntos; y actitudes liberales 0 erotofílicas de 12 a 15 puntos.

\section{Procedimiento}

Tras obtener los permisos necesarios de la comisión de ética de la Universitat de València, la persona responsable del proyecto contactó con los/as directores/as de los servicios de apoyo para personas con discapacidad intelectual de la Comunidad Valenciana, Castilla-La Mancha y Andalucía (España). Tras la exposición de los objetivos y el procedimiento del estudio, se concretó la agenda de evaluación con las instituciones que aceptaron participar. Seguidamente, varios miembros del equipo de investigación SALUSEX iniciaron la evaluación tras comprobar que los participantes cumplían los criterios exigidos. En el caso de las personas con DI, los criterios de inclusión fueron los siguientes: tener un grado de discapacidad leve o moderado, poseer suficientes capacidades verbales para responder a los cuestionarios y ser capaces de aceptar de forma libre y voluntaria la participación en el estudio. En el caso de los profesionales el criterio fue tener una experiencia trabajando con personas con DI de al menos 1 año en el mismo u otro centro ocupacional.

La evaluación de los usuarios se llevó a cabo en el consultorio del psicólogo de los centros ocupacionales donde acudían tanto los que vivían en la unidad familiar como quienes estaban en centros residenciales 0 
viviendas tuteladas. Paralelamente, Ios profesionales del centro que cumplían los criterios fueron evaluados por miembros del equipo SALUSEX.

\section{Análisis de datos}

Se realizaron análisis descriptivos (porcentajes) para establecer los niveles actitudinales y pruebas $t$ de Student para muestras independientes para analizar posible existencia de diferencias significativas entre las actitudes hacia la conducta sexual que tienen las personas con DI y las de los profesionales que trabajan con ellos. Todos análisis se realizaron con el paquete estadístico IBM SPSS 26.

\section{RESULTADOS}

Para comprar las respuestas dadas por las personas con DI y por los profesionales que trabajan con ellos se compararon tres ítems equivalentes de la Escala de Actitudes hacia la sexualidad en personas con discapacidad intelectual (Gil-Llario et al.) y la Escala de percepción sexual para personas con discapacidad intelectual (Browman et al., 1996). Los ítems seleccionados evaluaban actitudes relacionadas con tres prácticas sexuales: besos y caricias, sexo oral y coito vaginal.

El análisis de las respuestas proporcionadas acerca de las actitudes hacia la conducta sexual determina que un $91.7 \%$ de personas con DI y un $15 \%$ de los profesionales poseen actitudes moderadas/conservadoras, con una puntuación media de $8.36(\mathrm{DT}=12.16)$ y de $12.17(\mathrm{DT}=1.05)$, respectivamente, sobre un rango de 3 (actitudes completamente erotofóbicas) a 15 puntos (actitudes completamente erotofílicas). Analizando los rangos interpretativos con mayor detalle, el $36.4 \%$ de las personas con DI poseen actitudes conservadoras, el $55.3 \%$ actitudes moderadas y el $8.3 \%$ actitudes liberales. Por su parte, el $14 \%$ de los profesionales presentan actitudes moderas y el $86 \%$ actitudes liberales (ver Figura 1).

Figura 1. Actitudes hacia la conducta sexual según el nivel de erotofóbia/erotofilia

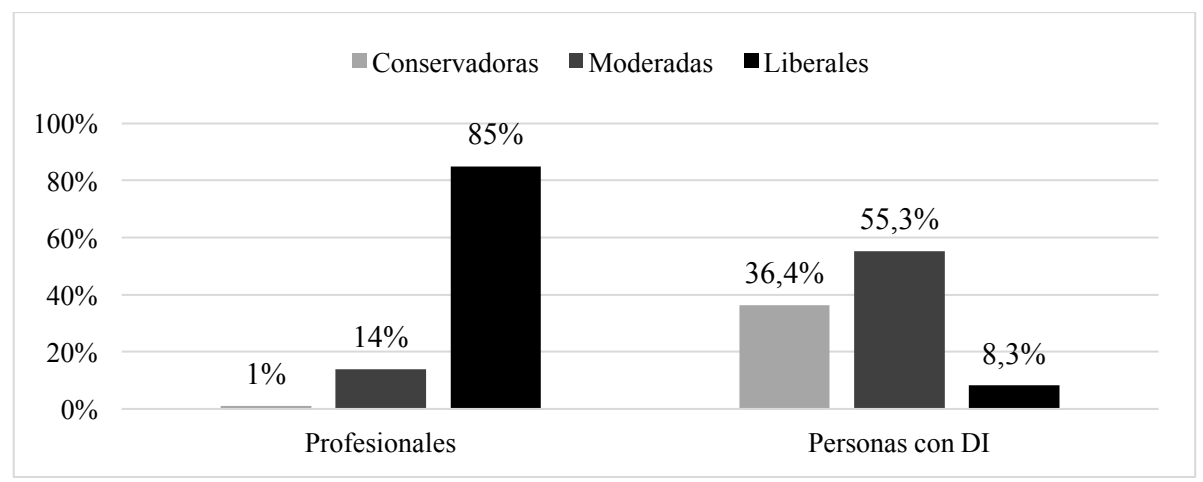

Examinando las diferencias actitudinales según el género del profesional (ver Figura 2) se observa que las mujeres presentan actitudes ligeramente más liberales con respecto a los hombres, aunque estas diferencias no resultan estadísticamente significativas ni en el sexo oral $(t=-1.221 ; p=.226)$ ni en el coito vaginal $(t=-.116$; $p=.908$ ). Las actitudes que presentan hombres y mujeres ante los besos y caricias son completamente liberales, obteniéndose en ambos grupos la máxima puntuación. 
Figura 2. Actitudes hacia la conducta sexual en función del género (profesionales)

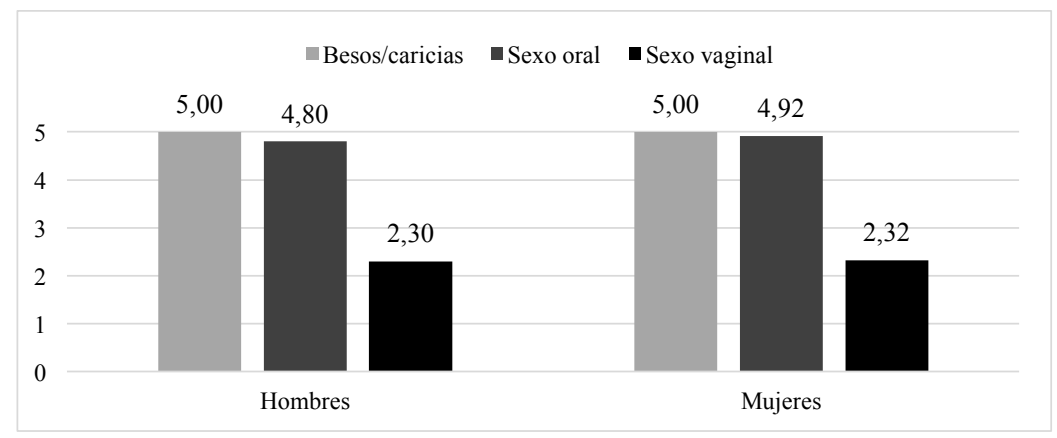

Figura 3. Actitudes hacia la conducta sexual en función del género (personas con DI)

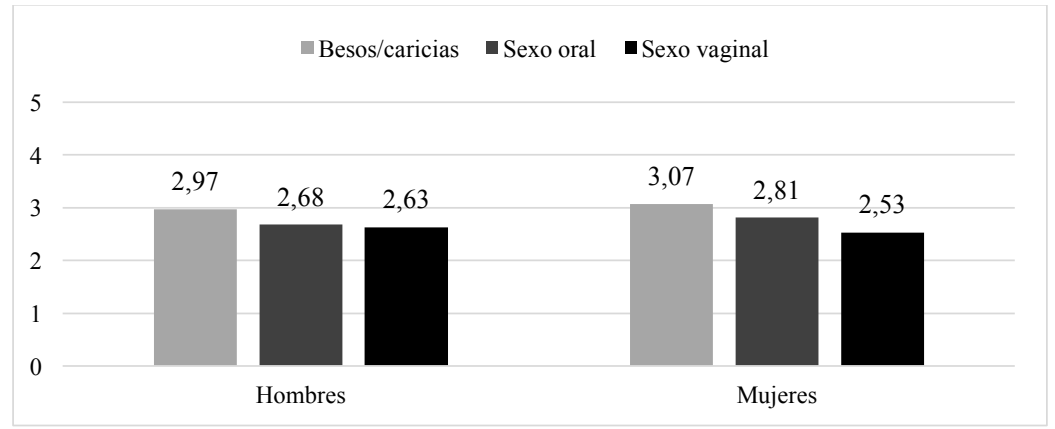

Los análisis estadísticos realizados en función del género entre hombres y mujeres con DI (ver Figura 3) establecen que las mujeres presentan actitudes más erotofílicas, pero sin alcanzar significación estadística, en los besos y caricias $(t=-.686 ; p=.493)$ y en el sexo oral $(t=-.971 ; p=.332)$. Por el contrario, los hombres refieren actitudes más liberales ante la práctica del coito vaginal (2.63 vs. 2.53), aunque estas diferencias tampoco resultan significativas $(t=.834 ; p=.405)$.

Las distintas pruebas de comparación de medias realizadas establecen que, en general, los profesionales presentan actitudes más liberales hacia la conducta sexual de las personas con discapacidad intelectual ( $\mathrm{M}=$ $12.17 ; \mathrm{DT}=1.05)$ que las propias personas que conforman este colectivo $(M=8.36 ; \mathrm{DT}=2.16)$, siendo estas diferencias estadísticamente significativas ( $t=-17.076 ; p=.001$ ). Esta diferencia presenta un tamaño del efecto grande $(d=2.25)$. Cuando se tiene únicamente en consideración el nivel más bajo de intimidad, «besos prolongados o abrazos", los resultados obtenidos van en la línea del hallazgo anterior. Así, los profesionales presentan actitudes más erotofílicas $(M=5 ; D T=0)$ que las personas con discapacidad intelectual $(M=3.02 ; D T=1.38)$, siendo estas diferencias nuevamente significativas $(t=-14.298 ; p=.001)$. El segundo nivel de intimidad « relaciones sexuales en las que no hay penetración » también revela actitudes congruentes con los resultados previos. Las actitudes que refieren los profesionales son más liberales ( $M=4.86$; $D T=.49)$ que las que poseen personas con DI ( $\mathrm{M}=2.75 ; \mathrm{DT}=1.25)$, alcanzándose una elevada significación estadística $(\mathrm{t}=-16.522 ; \mathrm{p}=.001)$. 
Figura 4. Actitudes hacia la sexualidad en función de la práctica sexual

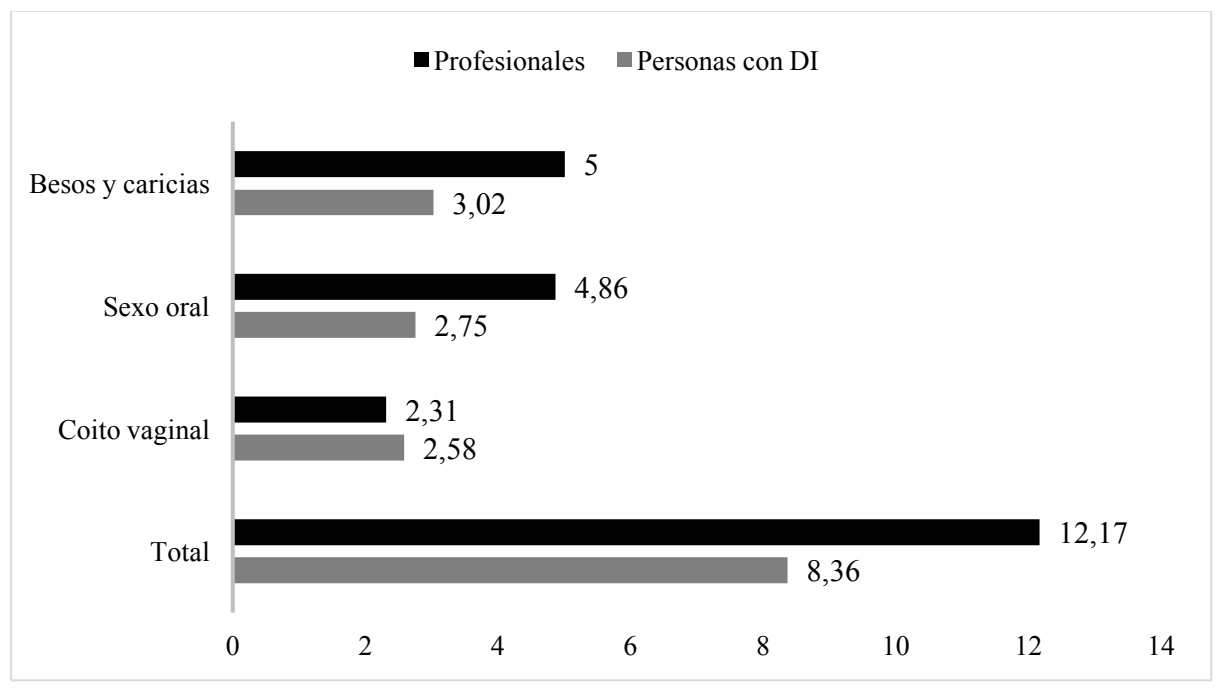

Finalmente, en el nivel de intimidad más elevado «relaciones sexuales en las que hay penetración» los resultados se invierten por completo. En este ítem son las personas con DI quienes presentan actitudes más erotofilicas $(\mathrm{M}=2.58 ; \mathrm{DT}=1.20)$ que las de los profesionales que trabajan con ellos $(\mathrm{M}=2.31 ; \mathrm{DT}=.86)$. Cabe destacar que, aunque esta diferencia es estadísticamente significativa $(t=2.107 ; p=.036)$, presenta un tamaño del efecto pequeño $(d=0.26)$.

\section{DISCUSIÓN}

El objetivo de este trabajo es analizar las actitudes hacia la conducta sexual en un grupo de personas con discapacidad intelectual y las de los profesionales que trabajan con ellos. Este tipo de estudios son importantes por diferentes razones. En primer lugar, las personas con DI han sido tradicionalmente consideradas personas asexuales 0 ajenas a la sexualidad, sufriendo una fuerte restricción a cualquier contenido de esta índole (Cobo, 2012). En segundo lugar, las actitudes mostradas por la sociedad son adoptadas por este colectivo e influyen en la perpetuación de diversos mitos y/o estereotipos relacionados con la sexualidad y la conducta sexual (Ditchman et al., 2017). Y, finalmente, los profesionales constituyen para las personas con DI una fuente de aprendizaje realista y no estereotipado (Siebelink et al., 2006).

La mayor parte de las personas con DI poseen actitudes conservadoras o moderadas, no obstante, dichas actitudes varían según la práctica sexual. Las actitudes más liberales quedan circunscritas a aquellas conductas que no implican penetración como, los besos, las caricias y, en menor medida, el sexo oral. La comunidad científica ha realizado pocos estudios donde se analicen las actitudes sexuales en las propias personas con discapacidad sexual. Normalmente son otras personas a quienes se les avalúan sus actitudes en relación a este colectivo (Eastgate, Scheermeyer, van Driel y Lennox, 2012; Franco, Cardoso y Neto, 2012). Los escasos datos existentes indican que, congruentemente con los hallazgos obtenidos, las personas con DI poseen actitudes generalmente conservadoras, que en algunos casos pueden ser moderadas (Díaz-Rodríguez et al., 2016). Este conservadurismo se deriva de las actitudes paternalistas, proteccionistas y condescendientes mostradas por gran parte de la sociedad (Murphy y Young, 2005; Swango-Wilson, 2009) y con las que estas personas se han socializado y crecido. En este sentido otros trabajos indican que, aunque la mayoría de las personas con DI aceptan las normas sexuales 


\section{¿HAY DIFERENCIAS ENTRE LAS ACTITUDES HACIA LA CONDUCTA SEXUAL QUE TIENEN LAS PERSONAS CON DISCAPACIDAD INTELECTUAL Y LAS DE LOS PROFESIONALES QUE TRABAJAN CON ELLOS?}

que se les atribuyen, otras empiezan a resistirse a ellas, cuestionando la visión estereotipada que la sociedad tiene (Azzopardi-Lane y Callus, 2015).

En general, las mujeres con DI poseen actitudes más liberales ante las caricias, Ios besos y el sexo oral, por el contrario, los hombres refieren actitudes más erotofílicas en la práctica del coito vaginal. Este aprendizaje, basado en los estereotipos de género, no difiere significativamente de la población sin discapacidad y está relacionado con los roles culturalmente construidos que designan las conductas y las funciones que se espera que los hombres y las mujeres adopten (Zaikman y Marks, 2014). La creencia de que la penetración es el aspecto central de la sexualidad es una idea todavía imperante entre muchos hombres (Herbenick et al., 2010). Por su parte, las mujeres presentan actitudes más favorables en todas las prácticas sexuales complementarias a la penetración bien por los estereotipos de género (Zaikman y Marks, 2014) o bien porque han sido instruidas para rechazar toda práctica que conlleve riesgo de embarazo (McDaniels y Fleming, 2016).

Los profesionales conforman un grupo con actitudes bastante liberales. Este dato es congruente con algunos estudios que indican que este colectivo rompe con los viejos estereotipos asociados a la sexualidad de las personas con DI (Brown y Pirtle, 2008), abogando por una educación sexual integral y adaptada a sus necesidades (Gil, Ballester, Caballero y Escalera, 2019). La formación de las personas con DI recae principalmente sobre este colectivo y sobre los padres. Los profesionales poseen una formación científica y actualizada que les permite tener unas actitudes más relistas hacia la sexualidad (Navarro, Torrico y López, 2010), mientras que los padres pueden mostrar sesgos basados en sus experiencias personales o en información estereotipada (Pownall, Jahoda y Hastings, 2012).

Los datos obtenidos no determinan diferencias entre las actitudes que poseen los profesionales hombres y las profesionales mujeres. En este sentido, la literatura científica establece que las diferencias de género no estarían presentes entre los propios profesionales, sino que se manifestarían en relación al género de las propias personas con DI (Pelleboer Gunnink, Van Oorsouw, Van Weeghel y Embregts, 2017). De este modo, los hombres con DI son percibidos como sujetos muy interesados por la sexualidad, mientras que las mujeres son percibidas como seres inocentes, que pueden ser fácilmente manipulables en las diversas facetas de la sexualidad (Young et al., 2012).

Las personas con DI poseen actitudes más erotofílicas hacia la práctica del coito vaginal que los profesionales. Este resultado confirma que algunos profesionales prefieren que las personas con DI no mantengan relaciones coitales, no porque esta práctica les parezca negativa en sí misma, sino por la posibilidad que acontezca un embarazo no deseado (Swango-Wilson, 2009). Algunos profesionales creen que este colectivo no posee capacidades suficientes para decidir sobre cuestiones tan importantes, como el uso de métodos anticonceptivos, por sí mismas (Olavarrieta et al., 2013).

En conclusión, los resultados obtenidos determinan la necesidad de mejorar las actitudes de las personas con DI, con el objetivo de conseguir la vivencia de una sexualidad libre, con suficiente información, que reduzca la adopción de comportamientos de riesgo. Para ello la implementación de programas educativos por parte de los profesionales, quienes a su vez han demostrado unas actitudes muy favorables hacia la sexualidad, puede constituir un aspecto muy positivo. En general, los profesionales constituyen modelos de aprendizaje significativo, así que, si se consigue que estos hablen sin tapujos ni restricciones de la diversidad afectivo-sexual, el cambio actitudinal tendrá mucha más garantía de éxito. No obstante, previamente a la implementación de estos programas, Ios profesionales deberían tener algunas sesiones formativas para tomar consciencia de sus actitudes y modificar aquellas que puedan interferir negativamente.

\section{AGRADECIMIENTOS}

Esta investigación ha sido realizada en el marco del proyecto de investigación "Desarrollo y análisis de la eficacia de un programa de educación afectivo-sexual para prevenir el abuso y mejorar la salud sexual en personas con diversidad funcional intelectual" financiado por el Programa Estatal de I+D+i Orientada a los Retos de la Sociedad del Ministerio de Ciencia e Innovación (Proyecto RTI2018-095538-B-100). 


\section{REFERENCIAS BIBLIOGRÁFICAS}

Álvarez, I., Vega, V., Spencer, H., González, F. y Arriagada, R. (2019). Adultos chilenos con discapacidad intelectual: creencias, actitudes y percepciones parentales sobre su autodeterminación. Siglo Cero, 50(2), 51-72. doi: 10.42201/scer020195025172

American Psychiatric Association (2013). DSM-5. Manual Diagnóstico y Estadístico de los Trastornos Mentales. Madrid: Panamericana.

Azzopardi-Lane, C. y Callus, A. M. (2015). Constructing sexual identities: people with intellectual disability talking about sexuality. British Journal of Learning Disabilities, 43(1), 32-37. doi:10.1111/bld.12083

Bandura, A. (2012). Self-efficacy: The exercise of control (12 ed.). New York: W.H. Freeman.

Bazzo, G., Nota, L., Soresi, S., Ferrari, L. y Minnes, P. (2007). Attitudes of Social Service Providers towards the Sexuality of Individuals with Intellectual Disability. Journal of Applied Research in Intellectual Disabilities, 20(2), 110-115. doi: 10.1111/j.1468-3148.2006.00308.x

Brown, R. D. y Pirtle, T. (2008). Beliefs of professional and family caregivers about the sexuality of individuals with intellectual disabilities: examining beliefs using a $Q$ methodology approach. Sex Education, 8(1), 59-75. doi: 10.1080/14681810701811829

Cezar, D. M., Figueiredo, V., Ferigato, S. y Agostini, R. (2016). Personas con discapacidad y sus roles ocupacionales: trabajo, familia, independencia y participación social. Revista Chilena de Terapia Ocupacional, 16(2), 107-117. doi:10.5354/0719-5346.2016.44755

Chou, Y. C., Jane, Z. Y. y Pu, C. Y. (2015). Attitudes Toward Male and Female Sexuality Among Men and Women with Intellectual Disabilities. Women \& Health, 55(6), 663-678. doi: 10.1080/03630242.2015.1039183

Cobo, C. (2012). Protocolo sobre relaciones interpersonales y sexualidad en personas con discapacidad intelectual, trastornos del espectro autista y otras discapacidades con déficit cognitivo usuarias de centros residenciales. Recuperado de http://www.juntadeandalucia.es/export/drupaljda/Personas_Discapacidad_Protocolo_sobre_relaciones_int erpersonales_y_sexualidad_pdint.pdf

Díaz-Rodríguez, I. (2017). Construcción de la salud sexual y análisis de su influencia en la calidad de vida y prevención de abusos sexuales en personas adultas con discapacidad intelectual. Tesis doctoral, Universitat de València, Valencia, España.

Díaz-Rodríguez, I., Gil-Llario, M. D., Morell-Mengual, V., Salmerón-Sánchez, P. y Ruiz-Palomino, E. (2016). Actitudes hacia la sexualidad: ¿difieren las personas con discapacidad intelectual de la población general? International Journal of Developmental and Educational Psychology, 1(1), 235-242. doi: 10.17060/ijodaep.2016.n1.v1.209

Ditchman, N., Easton, A. B., Batchos, E., Rafajko, S. y Shah, N. (2017). The Impact of Culture on Attitudes Toward the Sexuality of People with Intellectual Disabilities. Sexuality and Disability 35, 245-260. doi: 10.1007/s11195-017-9484-x

Eastgate, G., Scheermeyer, E., van Driel, M. y Lennox, N. (2012). Intellectual disability, sexuality and sexual abuse prevention: A study of family members and support workers. Australian Family Physician, 41(3), 135-139.

Fishbein, M. y Ajzen, I. (1975). Belief, attitude, intention and behavior: An introduction to theory and research. Reading, MA: Addison-Wesley.

Franco, D. G., Cardoso, J. y Neto, I. (2012). Attitudes Towards Affectivity and Sexuality of People with Intellectual Disability. Sexuality and Disability, 30, 261-287. doi: 10.1007/s11195-012-9260-x

Gil, M. D., Ballester, R., Caballero, L. y Escalera, C. (2019). Programa SALUDIVERSEX. Programa de educación afectivo-sexual para adultos con diversidad funcional intelectual. Madrid: Pirámide.

Gil-Llario, Fernández-García, Castro-Calvo, Caballero-Gascón y Ballester-Arnal (2020). Assesment of attitudes toward sexuality of people with intellectual disability. Manuscrito enviado para publicación. 
Gil-Llario, M. D., Morell-Mengual, V., Ballester-Arnal, R. y Díaz-Rodríguez I. (2018). The experience of sexuality in adults with intellectual disability. Journal of Intellectual Disability Research, 62(1), 72-80. doi: 10.1111/jir.12455

Herbenick, D., Reece, M., Schick, V., Sanders, S. A., Dodge, B. y Fortenberry, J. D. (2010). Sexual behavior in the united states: Results from a national probability sample of men and women ages 14-94. The Journal of Sexual Medicine, 7(S5), 255-265. doi: 10.1111/j.1743-6109.2010.02012.x

Kijak, R. (2013). The sexuality of adults with intellectual disability in Poland. Sexuality and Disability, 31(2), 109123. doi: /10.1007/s11195-013-9294-8

McDaniels, B. y Fleming, A. (2016). Sexuality education and intellectual disability: Time to ad-dress the challenge. Sexuality and Disability, 34(2), 215-225. doi: 10.1007/s11195-016-9427-y

Meaney-Tavares, R. y Gavidia-Payne, S. (2012). Staff characteristics and attitudes towards the sexuality of people with intellectual disability. Journal of Intellectual \& Developmental Disability, 37(3), 269-273. doi: 10.3109/13668250.2012.701005

Morell-Mengual, V., Gil-Llario, M. D., Díaz-Rodríguez, I. y Caballero-Gascón, L. (2017). Actitudes de padres, profesionales y población general hacia la sexualidad de las personas con discapacidad física e intelectual. International Journal of Developmental and Educational Psychology, 4(1), 173-183. doi: 10.17060/ijodaep.2017.n1.v4.1040

Murphy, N. y Young, P. C. (2005). Sexuality in children and adolescents with disabilities. Developmental Medicine and Child Neurology, 47(9), 640-644.

Navarro, Y., Torrico, M. E. y López, M. J. (2010). Programa de intervención psicosexual en personas con discapacidad intelectual. Educación y diversidad, 4(2), 75-92.

Olavarrieta, S., Darín, L., Suárez, P., Tur, N., Besteiro, B. y Gómez-Jarabo, G. (2013). Actitudes hacia la sexualidad, esterilización, maternidad/paternidad y habilidades de crianza de personas con discapacidad intelectual: Un estudio preliminar. Siglo Cero, 44(4), 55-69.

Organización Mundial de la Salud (2019). CIE-11. Clasificación Internacional de Enfermedades. Recuperado de https://icd.who.int/browse11/l-m/es

Pelleboer Gunnink, H. A., Van Oorsouw, W. M. W. J., Van Weeghel, J., \& Embregts, P. J. C. M. (2017). Mainstream health professionals' stigmatising attitudes towards people with intellectual disabilities: a systematic review. Journal of Intellectual Disability Research, 61(5), 411-434. doi: 10.1111/jir.12353

Pereira-Silva, N. L., Furtado, A. V. y Andrade, J. F. (2018). La Inclusión en el Trabajo desde la Perspectiva de las Personas con Discapacidad Intelectual. Trends in Psychology, 26(2), 1003-1016. doi:10.9788/tp2018.2$17 \mathrm{pt}$

Phasha, T. N. y Runo, M. (2017). Sexuality Education in Schools for Learners with Intellectual Disabilities in Kenya: Empowerment or Disempowerment? Sexuality and Disability, 35(3), 353-370. doi: 10.1007/s11195017-9480-1

Pownall, J. D., Jahoda, A. y Hastings, R. P. (2012). Sexuality and sex education of adolescents with intellectual disability: mothers' attitudes, experiences, and support needs. Intellectual and developmental disabilities, 50(2), 140-154. doi: 10.1352/1934-9556-50.2.140

Scotti, J. R., Slack, B. S., Bowman, R. A. y Morris, T. L. (1996). College student attitudes concerning the sexuality of persons with mental retardation: Development of the perceptions of sexuality scale. Sexuality and Disability, 14, 249-263. doi: 10.1007/BF02590098

Siebelink, E. M., de Jong, M. D. T., Taal, E. y Roelvink, L. (2006). Sexuality and People with Intellectual Disabilities: Assessmentof Knowledge, Attitudes, Experiences, and Needs. Mental retardation, 44(4), 283294. doi: 10.1352/0047-6765(2006)44[283:SAPWID]2.0.C0;2

Swango-Wilson, A. (2009). Perception of sex education for individuals with developmental and cognitive disability: A four cohort study. Sexuality and Disability, 27(4), 223-228. doi: 10.1007/s11195-009-9140-1 
Young, R., Gore, N. y McCarthy, M. (2012). Staff attitudes towards sexuality in relation to gender of people with intellectual disability: A qualitative study. Journal of Intellectual \& Developmental Disability, 37(4), 343-347. doi: 10.3109/13668250.2012.704983

Zaikman, Y. y Marks, M. (2014). Ambivalent Sexism and the Sexual Double Standard. Sex Roles 71, 333-344. doi: 10.1007/s11199-014-0417-1 
